\title{
Esophageal replacement by gastric transposition: A single surgeon's experience from a tertiary
}

\section{pediatric surgical centre}

Jake D Foster ${ }^{1}$, MD MRCS

Nigel J Hall ${ }^{1,2}$, PhD MRCPCH FRCS

S Charles Keys ${ }^{1}$, FRCS

David M Burge ${ }^{1}$, FRCS FRCPCH

1. Department of Paediatric Surgery and Urology, University Hospitals Southampton NHS Foundation Trust

2. University Surgery Unit, Faculty of Medicine, University of Southampton

\section{Corresponding Author:}

Mr Nigel Hall, University Surgery Unit, Faculty of Medicine, University of Southampton, Mailpoint

816, Southampton General Hospital, Tremona Road, Southampton. SO16 6YD

Phone: 02381206146

Email: n.j.hall@soton.ac.uk

Type of study: Treatment Study

Level of Evidence: IV 


\section{ABSTRACT}

\section{Background}

Many pediatric surgeons have limited experience of esophageal replacement. This study reports outcomes of esophageal replacement by gastric transposition performed by a single UK-based pediatric surgeon.

\section{Methods}

Consecutive patients were identified who underwent esophageal replacement by gastric transposition over a 28 year period. Clinical and demographic data were collected. Weight-for-age Zscores were calculated for esophageal atresia patients.

\section{Results}

Nineteen patients were identified. Indication in the majority was long-gap esophageal atresia $(n=17$; 10 with tracheo-esophageal fistula). At surgery, median age was 8.5 months (range 2-55), median weight was $7.4 \mathrm{~kg}$ (range 4.0-17.4kg). A right-sided thoracotomy or trans-hiatal approach was used. Median post-operative length of stay was 17.5 days (range 7-130), median intensive care stay was three days (range 1- 63). There were no deaths. Anastomotic leak rate at 30 days was $10.5 \%(n=2)$. One patient required early stricture dilatation. Median weight-for-age Z-score increased from -2.17 at one year of age to $-1.86,-1.70$ and -1.93 at 5,10 and 15 years.

\section{Conclusions}

Esophageal replacement by gastric transposition offers a potentially life-changing treatment however is associated with significant morbidity. The majority of patients eventually achieve full oral feeding and maintenance of weight gain trajectory. A right-sided approach to the esophagus is feasible. 
Keywords: Gastric transposition; Gastric pull-up; Esophageal atresia

Type of Study: Treatment Study

Level of Evidence: IV 


\subsection{INTRODUCTION}

\subsection{Background}

Esophageal replacement is a relatively rare procedure in the pediatric population, and many pediatric surgeons have limited experience of this surgery. The principal indication for esophageal replacement is long-gap esophageal atresia in situations where primary restoration of anatomical continuity is not feasible. Additionally, various other congenital and acquired disorders of the esophagus may occasionally necessitate esophageal resection and replacement.

A number of different techniques have been proposed for replacement of the esophagus, including gastric transposition, or interposition of a pedicled segment of small or large bowel [1]. Whilst each of these methods has their advocates, the paucity of published outcome data from these procedures makes comparison between methods difficult. At our tertiary pediatric surgery center, a single surgeon has performed all esophageal replacement operations since 1988, using a gastric-based conduit.

\subsection{Aim}

The aim of this study was to report peri-operative and longer-term outcomes following gastric transposition for replacement of the esophagus.

\subsection{METHODS}

\subsection{Patients and procedures}

A prospectively-maintained patient database was consulted to identify all patients who underwent esophageal replacement by gastric transposition at a UK tertiary care pediatric center between 1 January 1988 and 31 March 2017. All esophageal replacement operations at our unit during this time period were performed by one surgeon (DMB), either alone or in conjunction with a consultant colleague. 
The preferred surgical techniques adopted in these patients included initial formation of a cervical esophagostomy on the right side of the neck along with a feeding gastrostomy. Sham feeding was established as soon as possible. There were no specific age or weight criteria employed to dictate timing of esophageal replacement surgery but it was the senior author's impression that oral feeding was better tolerated if surgery was delayed until the infant was at least capable of sitting, in the knowledge that thoracic stomach emptying following this surgery is primarily by gravity. In most cases a surgical feeding jejunostomy was placed at the time of reconstruction and used until oral feeding had been established. It was intended that the surgical approach would avoid thoracotomy where possible but was felt to be appropriate when there had been a previous thoracotomy performed. Post-operative ventilation was anticipated in all cases but there was no predefined duration, extubation being undertaken when clinically appropriate. Patients were initially fed entirely via a jejunostomy or alternative enteral route, and oral intake was gradually introduced.

\subsection{Data extraction}

The study received local institutional approval as a retrospective evaluation of practice. Clinical notes were consulted for all included patients and data was extracted retrospectively from these. Data collected included patient demographics, indications for surgery, peri-operative clinical course, and clinical outcomes.

Length of stay and other post-operative timings were defined with the day of surgery considered to be day 0 . Weights were extracted from clinic notes, operating charts, drug charts and from medical and nursing documentation.

Anastomotic leakage was defined as confirmed extra-luminal passage of radiological contrast medium at the site of the anastomosis, with or without clinical symptoms of leakage. Radiological assessment of anastomotic integrity was not routinely performed prior to commencing oral diet, 
rather radiological evaluation was only performed if required based upon clinical assessment. Anastomotic stenosis was defined as a symptomatic anastomotic narrowing necessitating dilatation.

\subsection{Data analysis}

Continuous variables are presented as medians with range. Binary outcomes are presented as absolute number of cases.

Weight-for-age Z-scores (indicating the number of standard deviations from the mean weight at a specific age) were calculated using World Health Organization reference tables for child weights [2]. Z-scores are reported for weight on the date of surgery and for weights at birth, 1, 5, 10 and 15 years of age, as long as the weight was recorded within six months from these birthdays. The Z-scores were calculated for the exact age in months for on the date on which the weight was recorded, allowing accurate comparison amongst the patients. To facilitate a homogenous population for analysis, the two patients for whom the indication for surgery was not esophageal atresia were excluded from z-score analysis.

\subsection{RESULTS}

\subsection{Patients and indications for surgery}

Nineteen patients were identified who underwent gastric transposition for replacement of their esophagus at our center during the 28 year study period. An additional two patients were identified early in the study period who were treated with gastric tube graft formed from the greater-curve of stomach, with anastomoses to the cervical esophagus and to the gastro-esophageal junction [3]. These two patients were excluded from further analyses as they had not undergone gastric 
transposition surgery. Demographic details and indication for surgery for the nineteen patients are 


\subsection{Procedures}

The nineteen included patients underwent a gastric transposition procedure for replacement of their esophagus. For one of these patients gastric transposition was performed as a revision procedure following a gastric tube graft three months earlier where there was concern about the integrity of the anastomosis between tube graft and esophagus. This patient underwent thoracotomy and wash-out of a loculated pleural collection. Whilst no anastomotic leak was conclusively found, it was decided intra-operatively to remove the gastric tube and revise to a standard gastric transposition conduit.

All patients except the caustic injury patient were fed via gastrostomy prior to surgery. Fourteen patients had a cervical esophagostomy formed prior to gastric transposition surgery, allowing early establishment of milk sham feeds. This was performed on the right side of the neck as soon as it was decided that the gap was too long for primary repair. At surgery, median age was eight and a half months with a range of 2 - 55 months (the patient operated age 55 months was the caustic esophageal injury patient). Of the 17 patients with esophageal atresia, the oldest age at time of gastric transposition was 22 months: this was in a patient who had undergone primary anastomosis and had required multiple dilatations for anastomotic stricturing. Median weight at the time of surgery was $7.4 \mathrm{~kg}$, with range $4.0-17.4 \mathrm{~kg}$. Median operating time was five hours (range $3-10$ hours). Ten procedures were performed using a right sided thoracotomy together with abdominal and cervical incisions, whereas nine procedures were performed via abdominal and cervical incisions only. All eight patients who had previously undergone thoracotomy for ligation of fistula or attempt at primary repair required a thoracotomy approach to mobilize and resect the native esophagus, as did the patient with caustic injury and the patient with the congenital esophageal stricture. Where a throactomy was performed the gastric conduit was placed in the right side of the chest, whereas a posterior mediastinal location was applied when a trans-hiatal approach was used. Routine placement of thoracic or abdominal drains at the end of surgery was not performed. 
Sixteen patients had construction of a "Witzel" type feeding jejunostomy at the time of their gastric transposition surgery to facilitate post-operative feeding. The remaining three patients had nasojejunal feeding tubes passed across their anastomoses at the time of surgery rather than jejunostomy formation for post-operative enteral feeding.

\subsection{Peri-operative outcomes}

There were no peri-operative deaths. Median post-operative length of stay was 17.5 days (range 7130 days). Median length of post-operative intensive care stay was three days (range 1-63 days). All patients remained intubated in the immediate post-operative period for respiratory support. The median total length of time that patients remained intubated post-operatively was two days (range 1 - 51 days), including four patients who required re-intubation following an initial attempt at extubation.

Oral sips of water were first permitted at median post-operative day 8 (range $4-30$ ). The timing of progression to oral feeds was variable and dependent upon the patient's clinical picture. The overall anastomotic leak rate in this series at 30-days post-operation was two out of 19 patients $(10.5 \%)$ of whom one was treated entirely non-operatively with antibiotics and deferral of oral intake commencement. The second patient had a small, contained, peri-anastomotic collection detected 13 days post-operation which was also treated initially with antibiotics. This patient later required return to theatre to adjust their gastric conduit which was compressing the lung - although the anastomosis was left intact at this procedure. Ten patients had post-operative respiratory infections that were treated with antibiotics.

Two patients required post-operative placement of chest drains. In one patient the indication was a pneumothorax on day one post-op, whereas the second had a pleural drain placed on post-operative day five for drainage of a pleural effusion. 
Three patients required return to theatre during their index admission: one patient required replacement of a jejunostomy that became accidentally dislodged on post-operative day 25 , one patient required laparotomy and revision of their jejunostomy following the jejunum forming a volvulus around the jejunostomy, and one patient required abdominal mobilization of their gastric conduit which was causing lung compression as described above. No patients suffered graft necrosis following their surgery.

One patient required endoscopic dilatation of anastomotic stricture within the first six postoperative months: the first dilatation was performed at two months post-operation and this patient subsequently required one further dilatation.

\subsection{Long-term outcomes}

Median age at final follow-up assessment was 13 years of age (range $1-26$ years). None of the patients in this series have died during the follow-up period.

In addition to the patient that required early endoscopic dilatation of an anastomotic stricture as described above, one patient required a single dilatation of an anastomotic stricture three years on from their initial surgery, and the patient with the congenital stricture has required multiple dilatations of an anastomotic stricture during the follow-up period: the first of these dilatations was performed at three years after their operation.

Jejunostomy or nasogastric feeding was continued for median eight months post-operatively (range $1-162$ months). The majority of the patients (17/19) are now fully orally fed, with just two of the patients still receiving supplementary enteral tube feeding at the time of writing. One further patient experienced significant long-standing difficulties establishing oral intake, and required feeding via a jejunostomy up until thirteen years post-operatively - initially for all dietary intake, 
then for supplementary feeds. This patient underwent gastric transposition surgery age three months and had not established sham feeding effectively prior to surgery.

Two patients were reported during follow-up to be troubled by symptoms suggestive of dumping syndrome. One patient experienced episodes of flushing and paleness during the first postoperative year, however this spontaneously settled without need for intervention. The second patient was intermittently troubled from around three years post-operation and continued to experience difficulties for around seven years, including one period where naso-gastric feeding was required to deal with the dumping syndrome and supplement oral intake. Eventually this patient's symptoms were controlled with dietary manipulation and behavior modification.

Weight-for-age Z-scores were calculated for 16 of the patients who underwent gastric transposition for esophageal atresia (the most recently operated patient was less than one year on from their surgery at the time of writing and so was not included). Median weight-for-age Z-score at birth for these patients was -1.15 (range -2.76 to 0.91 ), and median Z-score at the time of surgery was -1.04 (range -2.46 to 0.40 ). At one year of age, median Z-score was -2.17 (range -4.03 to 0.86 ). At five years the median Z-score was -1.86 (range -3.41 to -0.24 ). Weights were available for 12 patients at age ten years, with median Z-score -1.70 (range -3.74 to 0.37 ). Weights were available for six patients at 15 years of age, with median Z-score -1.93 (range -2.76 to -0.94 ). These data are summarized in figure 1.

\subsection{DISCUSSION}

This study has reported upon a consecutive series of patients requiring esophageal replacement by gastric transposition over a 28 year period. Post-operative morbidity following this surgery is substantial, reflecting the hazardous nature of these procedures. In our series there was a $10 \%$ anastomotic leak rate and five children (26\%) required re-intervention for complications: one for 
lung compression, two for complications related to the jejunostomy, and two required placement of chest drains. Overall our peri-operative outcomes are similar to those reported in other series of patients. The largest series, authored by Spitz, reported upon 192 children operated upon at a quaternary pediatric surgery center in the UK from 1981, with $4.6 \%$ mortality, $12 \%$ anastomotic leakage rate, and $20 \%$ developing anastomotic stricture requiring dilatation [4].

There has been little long-term follow-up data published after gastric transposition surgery in infants and children. Our cohort of patients includes some who underwent their surgery over two decades ago. No children have died during follow-up. Some of the patients required prolonged nutritional supplementation, and two of the patients are still reliant upon supplemental tube feeding to meet their requirements. Long-term dietetic input and support is a universal requirement in this cohort of patients. Perhaps unsurprisingly, most patients were in the lower centiles for weight at the time of surgery, however they have managed to maintain their weight-for-age Z-score trajectory long-term indicating growth 'along' centile lines. We acknowledge that the data at 10 and 15 years of age are limited to a small number of patients. We also acknowledge that our study was a retrospective review, based upon clinical notes and outpatient letters and therefore some long-term information on feeding and other outcomes may not have been available. In particular it has been noted that long-term patients frequently suffer from iron-deficiency and chronic reflux [5]. At our institution we have not routinely performed endoscopic assessment of the remaining native esophagus and acknowledge this as a potential limitation of our data.

Our preferred approach for gastric replacement of the esophagus is to use a trans-hiatal approach via the posterior mediastinum without having to add the extra morbidity associated with a thoracotomy. However when a thoracotomy has been required we preferred a right-sided thoracic incision which gives improved access to the lower esophagus, rather than the left-sided thoracotomy incision used in most published descriptions of this technique $[6,7]$. We have shown that outcomes achieved using such an approach are similar to those reported in series using a left-sided approach, 
and this may represent a particularly useful learning point for surgeons planning their approach in a patient whose left thorax has previously been opened for cardiac surgery.

Fourteen of the patients in this study had formation of a right sided esophagostomy prior to their definitive esophageal resection. A right sided placement was used, facilitating future access via a right side thoracotomy incision. The formation of an esophagostomy enables the infant to establish early sham feeding without the risk of aspiration, and is recommended to enable satisfactory longterm oral intake after reconstruction is performed. In our series patients who did not undergo esophagostomy included those patients treated for non-atresia pathologies, and patients who underwent attempted primary repair for esophageal atresia.

Alternative conduits to the stomach have been reported for esophageal replacement, with both colon and jejunum interposition grafts widely used [8]. Gallo and colleagues undertook a systematic review of articles published in a five-year period and identified 15 studies within which $73 \%$ of patients had received colonic grafts, $21 \%$ gastric pull-up, and $6 \%$ jejunal interposition grafts [9]. They identified comparable peri-operative outcomes between colonic and gastric grafts, however concluded that proper prospective comparative studies are lacking, making accurate comparison between different methods imprecise at best. Other techniques use a gastric tube either proximally or distally based $[10,11]$. A gastric "tube" graft retaining the lower esophagus has also been described [12].

The current study does have a number of limitations. It represents clinical outcomes from a single surgeon's practice at a single center and the generalizability of our outcomes is questionable. However, there are only a small number of series published of this surgical technique, and our consecutive series represents one of the largest reported to date. Similarly, the time-period through which the patients in this study were operated upon stretched over 28 years, and operative and peri-operative practices are inevitably heterogeneous over such a large time-frame. A retrospective 
study such as this also relies upon accuracy of clinical documentation, and some subtleties of the clinical course may have been missed in some patients.

\subsection{Conclusions}

Esophageal resection and replacement using the stomach as a graft for treating long-gap esophageal atresia, caustic injury and other major esophageal disease is technically demanding with significant associated morbidity, even in experienced hands. Right sided esophagostomy and right thoracotomy, when needed, is a valid alternative to the traditionally reported left sided approach. Long-term these patients remain small for age, however have managed to maintain their weight gain trajectory throughout their follow-up. The data presented here can help surgeons counsel parents when considering this surgery. 
Table 1 - Demographic information for the included patients $(n=19)$

Figure 1 - Line graph showing median weight-for-age Z-scores at birth and at ages 1, 5, 10 and 15 for the 16 patients with esophageal atresia who underwent gastric pull-up for whom $>1$ year follow-up data was available. Error bars for each age point represent 1 standard deviation. 


\section{REFERENCES}

1. Sharma S, Gupta DK. Surgical techniques for esophageal replacement in children. Pediatr Surg Int. 2017; 33: 527-550.

2. http://www.who.int/childgrowth/standards/weight for age/en/ Last accessed $2^{\text {nd }}$ May 2017

3. Samuel M, Burge DM. Gastric tube interposition as an esophageal substitute: comparative evaluation with gastric tube in continuity and gastric transposition. J Pediatr Surg. 1999; 34: 264-269.

4. Spitz L. Gastric transposition in children. Semin Pediatr Surg. 2009; 18: 30-33.

5. Awad K, Jaffray B. Oesophageal replacement with stomach: A personal series and review of published experience. J Paediatr Child Health. 2017; 53: 1159-1166.

6. Spitz L. Gastric transposition via the mediastinal route for infants with long-gap esophageal atresia. J Pediatr Surg. 1984; 19: 149-154.

7. Spitz L, Coran A. Operative Pediatric Surgery, $7^{\text {th }}$ ed. Boca Raton, USA: CRC Press; 2013.

8. Loukogeorgakis SP, Pierro A. Replacement surgery for esophageal atresia. Eur J Pediatr Surg. 2013; 23: 182-190.

9. Gallo G, Zwaveling S, Groen H, et al. Long-gap esophageal atresia: a meta-analysis of jejunal interposition, colon interposition, and gastric pull-up. Eur J Pediatr Surg. 2012; 22: 420-425.

10. Heimlick HJ. Esophagoplasty with reversed gastric tube. Review of fifty three cases. Am J Surg. 1972; 123: 80-92.

11. Cohen DH, Middleton AW, Fletcher J. Gastric tube esophagoplasty. J Pediatr Surg. 1974; 9: 451-459.

12. Rao KL, Menon P, Samujh R, Chowdhary SK, Mahajan JK. Fundal tube esophagoplasty for esophageal reconstruction in atresia. J Pediatr Surg. 2003; 38: 1723-1725. 


\section{Figure 1}

Click here to download high resolution image

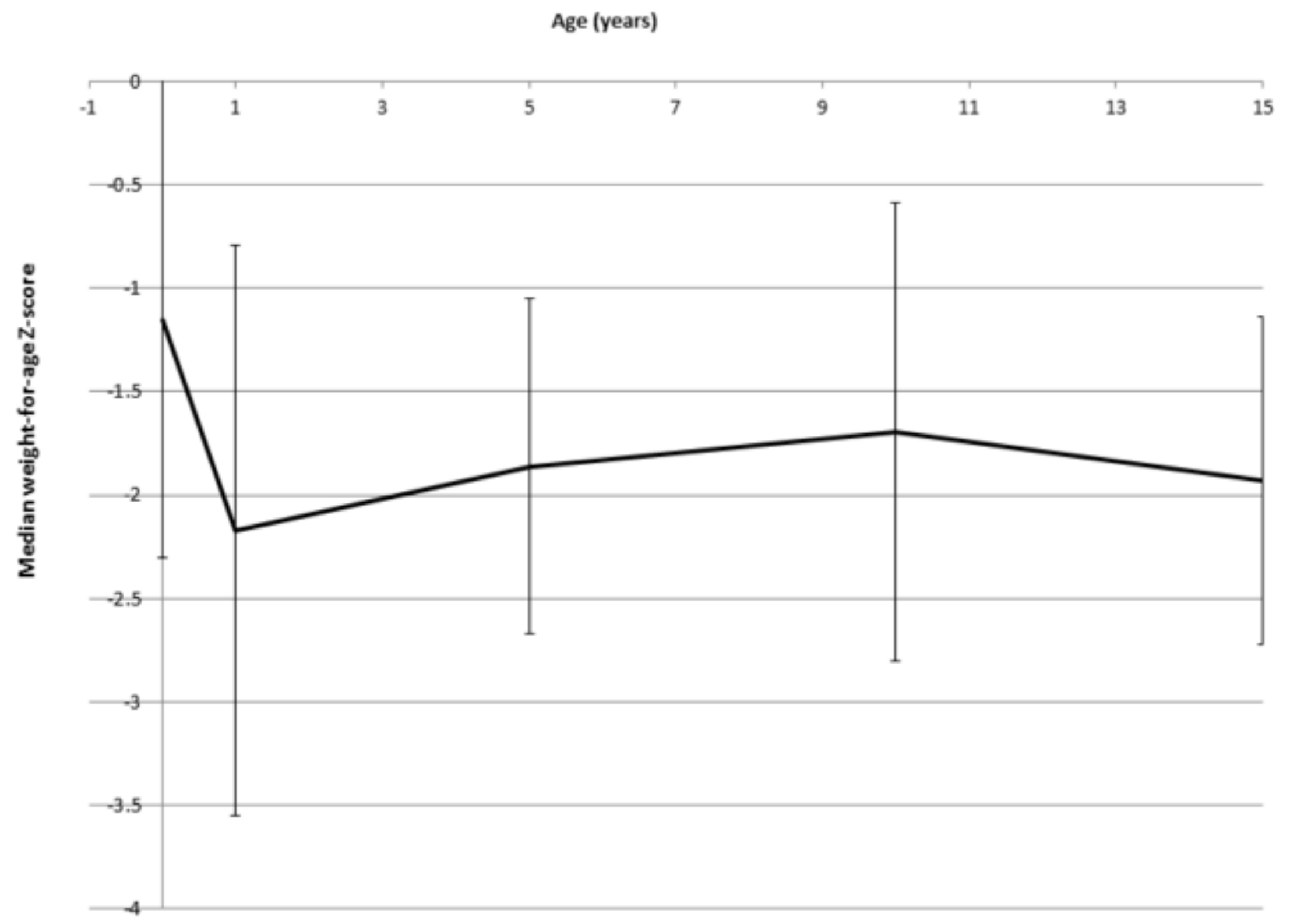




\begin{tabular}{|c|c|}
\hline $\begin{array}{l}\text { Gestational age at birth } \\
\text { (median, range) }\end{array}$ & 36.5 weeks ( 28 weeks -41 weeks) \\
\hline Birth weight (median, range) & $2.3 \mathrm{~kg}(1.1 \mathrm{~kg}-3.3 \mathrm{~kg})$ \\
\hline Gender & $\begin{array}{l}\text { Male. } n=11 \\
\text { Female. } n=8\end{array}$ \\
\hline $\begin{array}{l}\text { Indication for esophageal } \\
\text { replacement }\end{array}$ & $\begin{array}{l}\text { Esophageal atresia without fistula. } n=7 \\
\text { Esophageal atresia with upper pouch fistula. } n=5 \\
\text { Esophageal atresia with distal pouch fistula. } n=5 \\
\text { Caustic injury. } n=1 \\
\text { Extensive congenital stricture. } n=1\end{array}$ \\
\hline Major co-morbidity & $\begin{array}{l}\text { No major co-morbidity. } n=8 \\
\text { Anorectal malformation. } n=2 \\
\text { Renal developmental anomalies. } n=2 \\
\text { Large patent ductus arteriosus. } n=2 \\
\text { Tetralogy of Falot. } n=1 \\
\text { Other abnormal thoracic vascular anatomy. } n=1 \\
\text { Duodenal atresia \& anorectal malformation. } n=1 \\
\text { Encephalocele. } n=1 \\
\text { Sacral agenesis. } n=1\end{array}$ \\
\hline
\end{tabular}




\section{$\underline{\text { Journal of Pediatric Surgery Author Contribution Form }}$}

Study conception and design: Mr D Burge, Mr N Hall, Mr SC Keys

Data acquisition: Mr Jake Foster

Analysis and data interpretation: Mr Jake Foster, Mr D Burge, Mr N Hall, Mr SC Keys

Drafting of the manuscript: Mr Jake Foster

Critical revision: Mr D Burge, Mr N Hall, Mr SC Keys 